\title{
Estimation of parameters in heteroscedastic multiple regression model using leverage based near-neighbors.
}

\begin{abstract}
In this study, we propose a Leverage Based Near-Neighbor (LBNN) method where prior information on the structure of the heteroscedastic error is not required. In the proposed LBNN method, weights are determined not from the near-neighbor values of the explanatory variables, but from their corresponding leverage values so that it can be readily applied to a multiple regression model. Both the empirical and Monte Carlo simulation results show that the LBNN method offers substantial improvement over the existing methods. The LBNN has significantly reduced the standard errors of the estimates and also the standard errors of residuals for both simple and multiple linear regression models. Hence, the LBNN can be established as one reliable alternative approach to other existing methods that deal with heteroscedastic errors when the form of heteroscedasticity is unknown.
\end{abstract}

Keyword: Weighted least squares; Near-neighbors; Leverages; Monte Carlo simulation. 